\title{
Hyperspecral Skin Imaging with Artificial Neural Networks Validated by Optical Biotissue Phantoms
}

\author{
A. Bykov ${ }^{* 1}$, E. Zherebtsov ${ }^{1}$, V. Dremin ${ }^{1}$, A. Popov ${ }^{1}$, A. Doronin ${ }^{2}$, I. Meglinski ${ }^{1}$ \\ ${ }^{I}$ Opto-Electronics and Measurement Techniques Research Unit, University of Oulu,P.O. Box 4500, 90014 Oulu, Finland \\ ${ }^{2}$ School of Engineering and Computer Science, Victoria University of Wellington, PO Box 600, 6140, Wellington, New Zealand \\ *alexander.bykov@oulu.fi
}

\begin{abstract}
State-of-the-art micro-optic multichannel matrix sensor combined with the tunable Fabry-Perot micro interferometer enables a compact diagnostic device sensitive to the changes of the oxygen saturation as well as the blood volume fraction of human skin. The possibility of using Monte-Carlo modelling for neural network training in the problem of hyperspectral image processing has been demonstrated and validated using biotissue phantom and human skin in vivo. The proposed approach enables a tool combining both the speed of neural network processing and the accuracy and flexibility of Monte-Carlo modelling. (C) 2019 The Author(s)

OCIS codes: (170.0110) Imaging systems; (170.6510) Spectroscopy, tissue diagnostic; (110.4234) Multispectral and hyperspectral imaging
\end{abstract}

\section{Introduction}

Nowadays an intensive research is conducted in the field of biomedical application of hyperspectral imaging supported by recent progress in the hyperspectral instruments development and followed by their costs reduction. Potential applications of the considered technique are of high social impact and include monitoring and diagnostics of diabetic ulcer formation, rheumatic complications, wound healing control, diagnosis of melanoma and other malignancies.

Here we present a new approach based on the application of Artificial Neural Networks (ANN) to hyperspectral imaging for quantitative functional diagnostics of skin including 2D mapping of skin blood distribution (skin perfusion) and its oxygen saturation. For the validation of the proposed method we have developed a calibration phantom with predefined optical properties mimicking human bloodless dermis with the embedded capillaries filled with human blood of known oxygen saturation.

\section{Methods and Materials}

The imaging device was constructed on the basis of the hyperspectral snapshot camera (Senop Optronics, Finland) utilizing a Fabry-Perot tunable filter providing spectral resolution of 8-12 nm within the range of 500-900 nm. A broadband illumination unit utilizing 50W halogen lamp is based on the fiber-optic ring illuminator combined with the camera providing uniform distribution of light intensity in the camera field of view. The illumination ring and the camera are equipped with the rotatable broadband linear polarizers used for reduction of specular reflection from the skin surface. Generally, the constructed device is capable of recording spatially and spectrally resolved skin reflectance (hypercube) used for further ANN-based analysis.

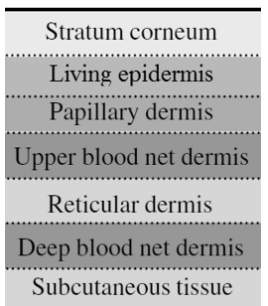

(a)

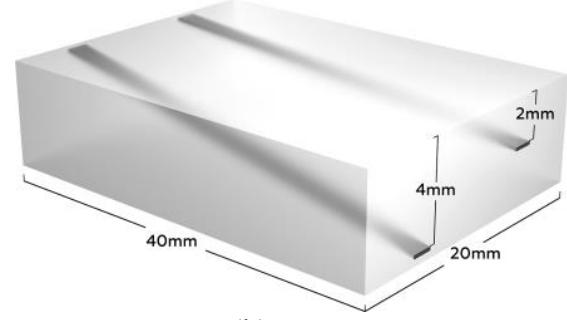

(b)

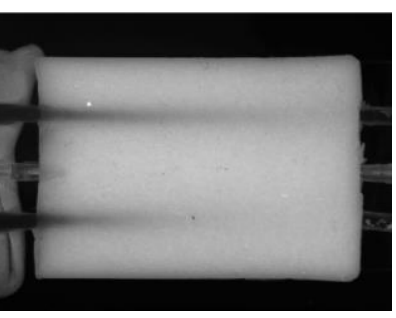

(c)

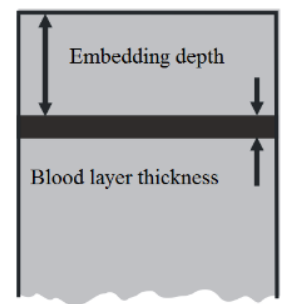

(d)

Fig. 1. 7-layer skin model used for calculations of diffuse reflectance spectra (a); 3D schematics of the biotissue phantom with the embedded blood vessels (b); photograph (top view) of the manufactured phantom with the channels filled with fully oxygenated blood (c); 3-layer tissue phantom model used for calculations of diffuse reflectance spectra.

The quantitative assessment of major physiological skin parameters was performed with the specially developed ANN algorithm. For the training of ANN a set of diffuse reflectance spectra of human skin simulated by the inhouse developed Monte Carlo (MC) method has been used extensively. The volume fraction of blood, oxygen saturation, melanin content and thickness of the epidermal layer were used as the variable parameters in the utilized seven-layer MC-based skin model (see Fig. 1a). The total training set contained 82,005 spectra in the range of 505$900 \mathrm{~nm}$ simulated with a step of $5 \mathrm{~nm}$. To validate the proposed method a biotissue phantom with the predefined 
optical properties equal to those of human dermis has been created. The detailed description of manufacturing and characterization of biotissue phantoms is given in $[1,2]$. The layout and the dimensions of the developed phantom are shown in Fig. 1b, c. The phantom contains two tilted plain hollow channels $(0.25 \times 1 \mathrm{~mm}$ cross-section $)$ located at the different angles inside the phantom. The embedding depth linearly increases from 0.3 to $2 \mathrm{~mm}$ for top channel and from 0.3 to $4 \mathrm{~mm}$ for the deep one. Fully oxygenated human blood from healthy donor was pumped through the channels with the rate of $10 \mathrm{ml} / \mathrm{h}$ to avoid blood sedimentation. For the training of ANN and estimation of blood oxygen saturation, the diffuse reflectance spectra were simulated by MC technique using 3-layer tissue model (Fig. 1d) accounting the blood layer embedding depth and its oxygen saturation as the variable parameters. Totally 11,628 spectra have been obtained.

\section{Results and conclusion}

The developed imaging system has been successfully used to perform the occlusion test measurements with healthy volunteers of different gender and skin color. Fig. 2a, b shows the results of the recovered blood volume fraction in skin and oxygen saturation level during the ring finger occlusion. It is seen that the occluded finger has significantly lower blood perfusion and oxygenation.

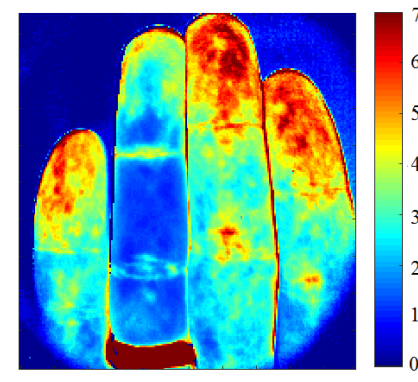

(a)

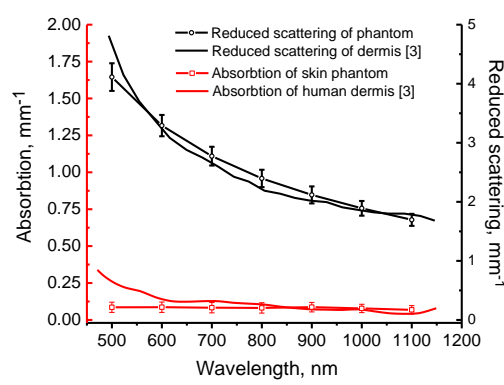

(d)

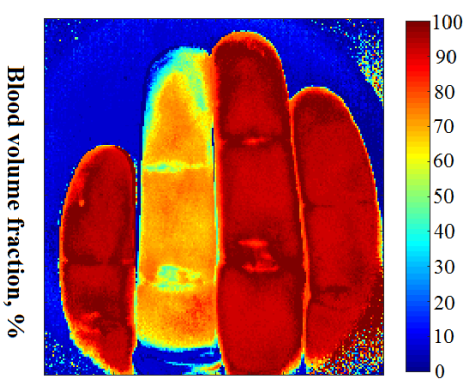

(b)

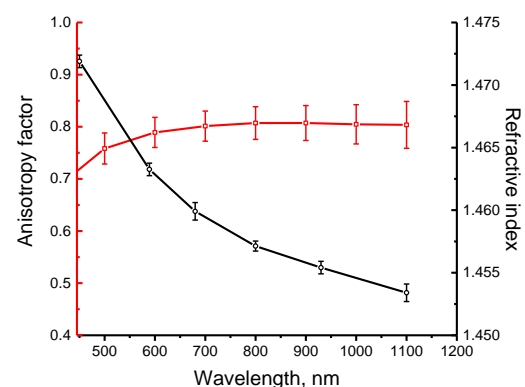

(e)

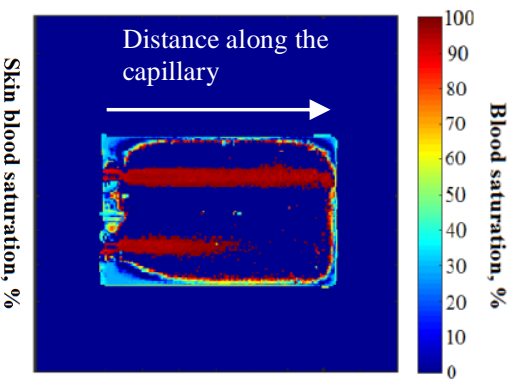

(c)

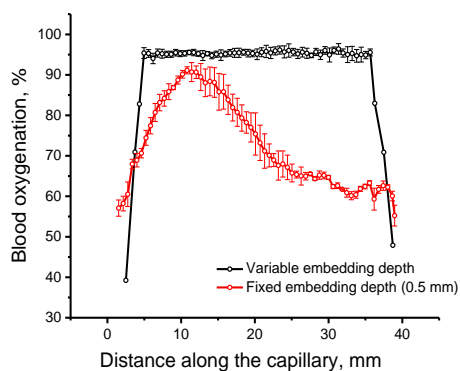

(f)

Fig. 2. 2D maps of blood volume fraction (a) and skin blood oxygenation (b) during ring finger occlusion; 2D map of blood oxygen saturation in the manufactured phantom (c); measured optical properties of the biotissue phantom (d,e); recovered values of blood oxygen saturation along the upper channel in the biotissue phantom (f).

The results of the phantom optical properties characterization (Fig. 2d, e) show good correspondence of the reduced scattering and absorption with those of human dermis in the range of 700-1100 nm. Fig.2c shows the recovered blood oxygen saturation along the embedded channels in the tissue phantom. It is seen that the correct value of blood oxygenation in this case can be recovered up to the depth of about $2 \mathrm{~mm}$. Further increase of the embedding depth causes the immediate signal loss. This result was obtained accounting the dependence of the simulated spectra on the channel embedding depth. However, the application of the numerical model with the fixed embedding depth (e.g. $0.5 \mathrm{~mm}$ ) leads to the underestimation of recovered blood saturation (Fig. $2 \mathrm{f}$, red curve). The correct value is obtained only for the embedding depth corresponding to that in the numerical model. This result highlights the importance of correct tissue model selection for the accurate measurements of tissue parameters.

\section{Acknowledgement}

Authors acknowledge the support of the Academy of Finland (grants: 290596, 296408).

\section{References}

[1] M. Wrobel, A. Popov, A. Bykov, et al., "Measurements of fundamental properties of homogeneous tissue phantoms", J. Biomed. Opt. 20(4), 045004 (2015).

[2] M. Wrobel, A. Popov, A. Bykov, et al., "Multi-layered tissue head phantoms for noninvasive optical diagnostics", J. Innov. Opt. Health Sci., 8(3), 1541005 (2015).

[3] E. Salomatina. et al., "Optical properties of normal and cancerous human skin in the visible and near-infrared spectral range," J. Biomed.

Opt., 11 (6), 064026 (2006). 